\title{
Identification and characterization of microRNAs in raw milk during different periods of lactation, commercial fluid, and powdered milk products
}

Xi Chen ${ }^{1, *}$, Chao Gao ${ }^{1, *}$, Haijin $\mathrm{Li}^{1, *}$, Lei Huang ${ }^{1}$, Qi Sun ${ }^{1}$, Yanye Dong ${ }^{1}$, Chunliang Tian ${ }^{2}$, Shengpu Gao ${ }^{3}$, Hailin Dong ${ }^{2}$, Danping Guan ${ }^{2}$, Xiaoyun $\mathrm{Hu}^{2}$, Shujian Zhao ${ }^{2}$, Liang $\mathrm{Li}^{1}$, Lin Zhu ${ }^{1}$, Qiao Yan ${ }^{1}$, Junfeng Zhang ${ }^{1}$, Ke Zen ${ }^{1}$, Chen-Yu Zhang ${ }^{1}$

${ }^{I}$ Jiangsu Diabetes Center, State Key Laboratory of Pharmaceutical Biotechnology, School of Life Sciences, Nanjing University, 22 Hankou Road, Nanjing 210093, China; ${ }^{2}$ Nanjing MicroMedMark Biotech Co., Ltd., 88 Zhujiang Road, Nanjing 210008, China; ${ }^{3}$ China National Institute of Standardization, ZhiChun Road, HaiDian District, Beijing 100088, China

Recent baby formula milk powder contamination incidents have shown that the classic markers or standards in milk quality control are insufficient in identifying "manipulated" poor-quality milk. In the present study, we demonstrated for the first time that cow milk contains large amounts of microRNAs (miRNAs) and that the unique expression profile of milk-specific miRNAs can serve as a novel indicator and possible new standard for the quality control of raw milk and milk-related commercial products, such as fluid milk and powdered formula milk. First, using Solexa sequencing, we systematically screened miRNA expression in raw milk and identified a total of 245 miRNAs in raw milk. Unlike other classic biomarkers whose expression levels are nearly identical at different periods of lactation, individual miRNAs can be significantly altered during lactation process, implicating that miRNAs may be a more accurate indicator to reflect the quality alteration of milk. Second, using TaqMan probe-based miRNA quantitative RT-PCR, we further identified seven miRNAs that have a relatively consistent expression throughout the lactation process, and more importantly, the expression profile of these seven milk-specific miRNAs can serve as an ideal biomarker for discriminating poor-quality or "manipulated" milk from pure raw milk, as well as for the quality control of commercial milk products, such as fluid milk and powdered formula milk. Together, our findings provide a basis for understanding the physiological role of milk miRNAs and a new potential standard for determining the quality of raw milk or milk-related commercial products.

Keywords: microRNA; milk; fluid milk; powdered milk

Cell Research (2010) 20:1128-1137. doi:10.1038/cr.2010.80; published online 15 June 2010

\section{Introduction}

Milk feeding is the most essential process underlying the foundation of human health during postnatal development [1]. Milk and milk products contain a good balance

\footnotetext{
*These three authors contributed equally to this work. Correspondence: Chen-Yu Zhang ${ }^{\mathrm{a}}$, Ke Zen ${ }^{\mathrm{b}}$, Junfeng Zhang ${ }^{\mathrm{c}}$

Tel/Fax: +86-25-83686234

${ }^{a}$ E-mail: cyzhang@nju.edu.cn

${ }^{b}$ E-mail: kzen@nju.edu.cn

${ }^{c}$ E-mail: jfzhang@nju.edu.cn

Received 9 April 2010; revised 14 May 2010; accepted 14 May 2010; published online 15 June 2010
}

of protein, fat, and carbohydrate and are very important sources of essential nutrients, including minerals, vitamins, riboflavin, pantothenic acid, and others [1]. However, the lack of accurate and efficient biomarkers for detecting the raw milk content presents a daunting task for milk quality control. Although the current markers for milk quality control, including the amounts of protein, lipid, carbohydrates, or ions, are quite useful in forming various industry standards, these individual markers are relatively easy to be manipulated and are therefore difficult to be used in monitoring the actual milk content. For example, melamine was found to be added to certain sub-standard or diluted milk to make the protein levels appear higher $[2,3]$. Although stringent inspection can 
effectively detect the contamination by melamine, other toxic materials might be added to the unqualified milk products to meet the "standard", which would still pose a great danger for human health. The problem of "manipulated" milk with poor quality cannot be solved unless there is a novel biomarker that not only fully reflects the quality of raw milk, but is also difficult to be manipulated.

Recently, a new class of RNA regulatory genes known as microRNAs (miRNAs) has been found to introduce a whole new layer of gene regulation in eukaryotes [4-6]. miRNAs are endogenous non-coding RNAs, consisting of 19 to 24 nucleotides in length. They play an important role in regulating gene expression by base-pairing to the complementary sites on the target mRNAs, thus blocking the translation or triggering the degradation of the target mRNAs [4-6]. Recent studies by our group and others have shown that miRNAs are stably present in many body fluids, including serum [7-10], plasma [7, 8], saliva [11], urine [12], and cell culture supernatants [13, 14], and that the unique expression patterns of miRNAs in various body fluids may serve as non-invasive or minimally invasive biomarkers for various diseases [7-12]. In the present study, we extended our previous work on circulating miRNAs and established an miRNA-based biomarker for milk quality control. Specifically, we showed that miRNAs are highly expressed in raw milk. Seven milk-associated miRNAs, miR-26a, miR-26b, miR-200c, miR-21, miR-30d, miR-99a, and miR-148a, were identified as potential biomarkers for the quality control of raw milk and other milk-related products, such as commercial fluid milk, infant formula powdered milk, etc.

\section{Results}

Genome-wide miRNA expression profiling in cow milk at different lactation stages

To test whether miRNAs are stably present in raw milk, we first used the Solexa sequencing technique to construct a library for all small RNAs from normal raw milk. As a high-throughput sequencing technology producing highly accurate, reproducible, and quantitative readouts of small RNA expression, Solexa can detect and define all RNA molecules including miRNA with various expression levels, and can unambiguously distinguish miRNAs from other small RNAs by referencing to various established RNA libraries [7, 15]. Using this method, we detected a total of 2487394 reads for mature milk

Table 1 The sequencing frequencies of the tissue-specific miRNAs in mature milk and colostrum

\begin{tabular}{|c|c|c|c|}
\hline Expression patterns & Name & Solexa reads in mature milk & Solexa reads in colostrum \\
\hline \multirow[t]{3}{*}{ Brain-specific [18-23] } & miR-9 & 1 & 0 \\
\hline & $\operatorname{miR}-124 a$ & 0 & 0 \\
\hline & miR-219-5p & 0 & 8 \\
\hline Sensory organ-specific $[18,24]$ & $\operatorname{miR}-96$ & 22 & 45 \\
\hline \multirow[t]{4}{*}{ Muscle-specific [20-22] } & miR-1 & 16 & 14 \\
\hline & miR-133a & 0 & 1 \\
\hline & miR-206 & 16 & 9 \\
\hline & $\operatorname{miR}-208$ & 0 & 0 \\
\hline Endothelial-specific [27] & $\operatorname{miR}-126$ & 0 & 0 \\
\hline Blood cell-specific [28] & $\operatorname{miR}-451$ & 2 & 25 \\
\hline \multirow[t]{6}{*}{ Immune cell/organ-specific [17] } & miR-155 & 110 & 121 \\
\hline & miR-181a & 1023 & 6255 \\
\hline & miR-142-5p & 69 & 204 \\
\hline & $\operatorname{miR}-223$ & 407 & 5650 \\
\hline & miR-146a & 4 & 2 \\
\hline & $\operatorname{miR}-150$ & 123 & 1232 \\
\hline
\end{tabular}


and 3398788 reads for colostrum, respectively (Supplementary information, Table S1). All RNAs sequenced by Solexa are in the length of 18-30 nt. Subsequently, those small RNAs were classified into different categories, according to their biogenesis and annotations. As shown in Supplementary information, Table S1, both mature milk and colostrum contain several heterogeneous small RNA species, including miRNAs, and degraded rRNA fragments and mRNA fragments. In milk, miRNAs are the major fraction of small RNA species. After classification, we found that the total reads of miRNAs in mature milk are similar to that in colostrum (1 418136 reads in mature milk vs. 1594965 reads in colostrum, Supplementary information, Table S1).

To further investigate the expression profile of miRNAs in raw milk, we compared the miRNAs identified in mature milk to those identified in colostrum. By referencing to the miRBase release 13.0 [16], Solexa sequencing identified 213 and 230 known miRNAs in mature milk and colostrum, respectively (Supplementary information, Table S2). The sequencing frequencies of these miRNAs are detailed in Supplementary information, Table S3. As shown in Figure 1A and Supplementary informa- tion, Table S3, most of the miRNAs were detected in both colostrum and mature milk, whereas only a small number of miRNAs were exclusively present in either colostrum or mature milk. Colostrum-specific miRNAs included miR-18a, miR-19a, miR-140-5p, miR-219$5 \mathrm{p}$, etc., while miR-10b, miR-9, etc. represented mature milk-specific miRNAs. Subsequently, we performed further analysis to select a group of miRNAs that are differentially expressed between mature milk and colostrum (detailed criteria are listed in the Materials and Methods section). Our analysis identified 116 miRNAs that were differentially expressed between colostrums and mature milk, in which 108 miRNAs were up-regulated and 8 miRNAs were down-regulated in colostrum compared to mature milk (Supplementary information, Table S4). In other words, the miRNA concentration in mature milk is decreased compared to that in colostrum. These results suggest that the composition of milk miRNAs is dynamically altered during the lactation process. However, in contrast to the significant decrease in miRNA concentration from colostrum to mature milk, the protein levels in colostrum and mature milk were nearly identical (Supplementary information, Table S5).
A

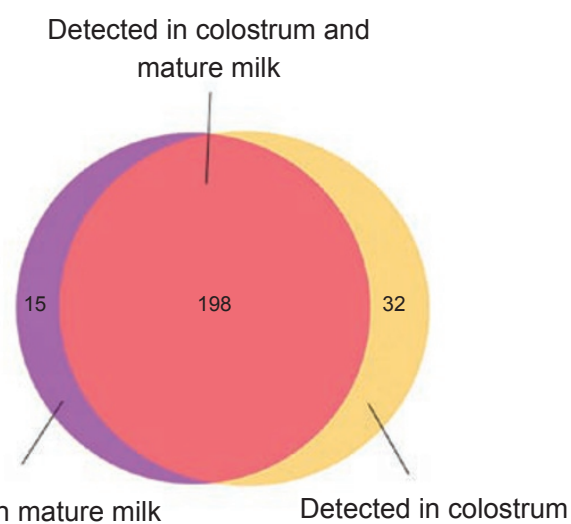

Detected in mature milk

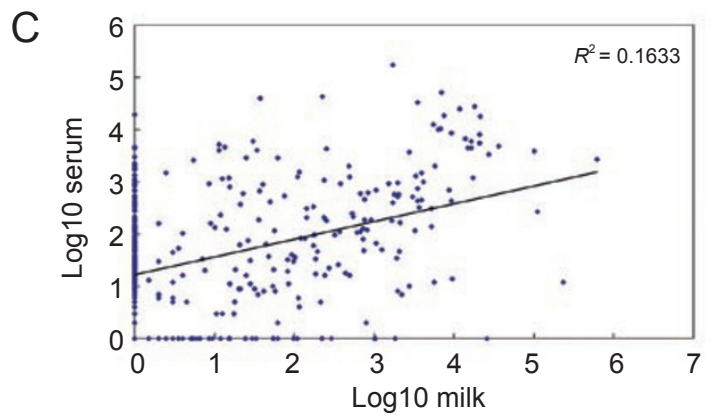

B

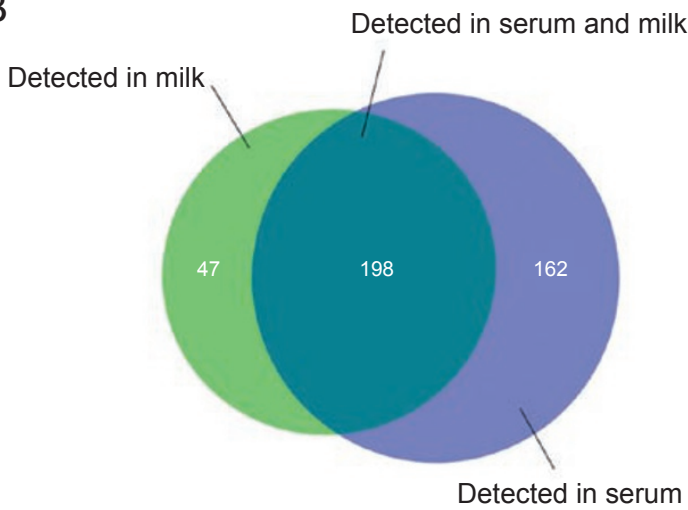

Figure 1 Characterisation of miRNAs in raw milk. (A) Number and overlap of miRNAs between mature milk (9 months postpartum) and colostrum (7 days postpartum). (B) Number and overlap of miRNAs between raw milk and serum. (C) Pearson correlation scatter plot of miRNA levels in raw milk and serum $\left(R^{2}=0.1633\right)$. 
Another interesting observation was that all of the miRNAs involved in immune response and immune system development, such as miR-181a, miR-155, and miR223 [17], were abundantly expressed in milk, especially in colostrum (Table 1). In contrast, tissue-specific miRNAs, such as miR-9/124a (brain) [18-23], miR-183/184 (sensory organ) [18, 24], miR-1/133 (muscle) [20-22], miR-216/217 (pancreas) [25], miR-122 (liver) [20-22, 26], miR-126 (endothelial) [27], and miR-451 (blood cell) [28], were generally less expressed in colostrum and mature milk. Although the molecular basis underlying this phenomenon remains to be shown, it is intriguing to speculate that a pool of such immune-related miRNAs may play a critical role in the biogenesis and development of immune system in infants. In this regard, the reduction of these miRNAs in mature milk compared to colostrum was in concert with the requirement of infant development.

\section{Identification of milk-specific or milk-enriched miRNAs}

To identify milk-specific or milk-enriched miRNAs, we assessed miRNA expression profile of the serum samples from healthy cows using the same strategy and compared the miRNA expression profile of cow serum with that of raw milk. In this comparison, the mean expression levels of miRNAs in colostrum and mature milk were used to represent general miRNA levels in raw milk. As shown in Supplementary information, Table S6, although the protein concentration was about three times lower in raw milk than in serum (serum: $60-80 \mathrm{~g} / \mathrm{L}$ vs. milk: 25 $35 \mathrm{~g} / \mathrm{L}$ ), the total amount of miRNAs in milk was about two-fold higher. Solexa sequencing and RNA classification indicated that expression profiles of miRNAs in serunh and milk are significantly different. As shown in Figure 1B, there were 47 miRNAs that were expressed in milk but were missing in serum, while serum contained 162 new miRNAs compared to milk. As shown in the Pearson correlation scatter plot (Figure 1C), the Pearson correlation coefficient $(R)$ between milk miRNAs and serum miRNAs was quite low. The significant difference between milk miRNA profile and serum miRNA profile is summarised in Supplementary information, Table S7. Using the same criteria of differentially expressed miRNA selection, including the copy number $>20$ and fold- change $>2$, we identified 105 miRNAs that were milkspecific or milk-enriched compared to miRNA expression in serum (Supplementary information, Table S8).

\section{Seven milk-specific miRNAs as a marker for quality con-} trol of milk

To establish an miRNA-based indicator for determining milk quality, we selected seven miRNAs that had a relatively high and consistent expression in raw milk at various lactation periods (Solexa reads $>3000 ; 0.5<$ colostrum/mature milk $<2$ ) as potential markers to be further validated. The Solexa reads of these seven miRNAs, including miR-26a, miR-26b, miR-200c, miR-21, miR30d, miR-99a, and miR-148a, are shown in Table 2. Subsequently, the expression levels of these seven miRNAs in raw milk collected at various stages of lactation (day 7 , month 1 , month 5, and month 9) were assessed by a TaqMan probe-based qRT-PCR assay [29, 30]. To accurately determine the absolute expression levels of the selected miRNAs, a series of synthetic miRNA oligonucleotides of known concentrations were also reverse-transcribed and amplified to construct a standard curve. The resulting $C_{\mathrm{T}}$ values were plotted vs. the $\log _{10}$ of the amount of input miRNAs. As shown in Figure 2A, decreasing the miRNA input led to a corresponding increase in the mean $C_{\mathrm{T}}$ values with a Pearson correlation coefficient $(R)$ close to 1 , suggesting that the resolution of our qRT-PCR assay is enough to measure the absolute level of miRNAs in a wide range of concentrations. The absolute amount of each miRNA in milk was then calculated by referring to the standard curve. As shown in Figure 2B, the expression levels of the seven miRNAs in milk from different stages of lactation were quite consistent, implicating the use of the expression profile of these miRNAs as an internal marker for milk quality. In addition, the expression levels of these seven miRNAs in raw milk were quite high, ranging from 0.1 to $10 \mathrm{pM}$ (Figure 2C), while their concentrations in cow serum were extremely low (Figure 2D). Serving as a control, the total protein levels in milk from different stages of lactation were identical (Figure 2E). Taken together, these results suggest that the expression levels of these seven miRNAs in milk are consistent during various stages of lactation and are coherent with the protein levels of raw milk.

Table 2 The sequencing frequencies of the seven miRNAs in mature milk, colostrum, and serum

\begin{tabular}{lccccccc}
\hline & miR-26a & miR-26b & miR-200c & miR-21 & miR-30d & miR-99a & miR-148a \\
\hline Mature milk & 3376 & 6595 & 11010 & 24137 & 14089 & 24097 & 16597 \\
Colostrum & 4855 & 3906 & 7996 & 47570 & 18922 & 17151 & 11298 \\
Serum & 990 & 141 & 14 & 4842 & 4405 & 5534 & 6601 \\
\hline
\end{tabular}

Total sequencing frequencies of miRNAs in mature milk, colostrum, and serum are 1418 136, 1594 965, and 660 162, respectively. 
A

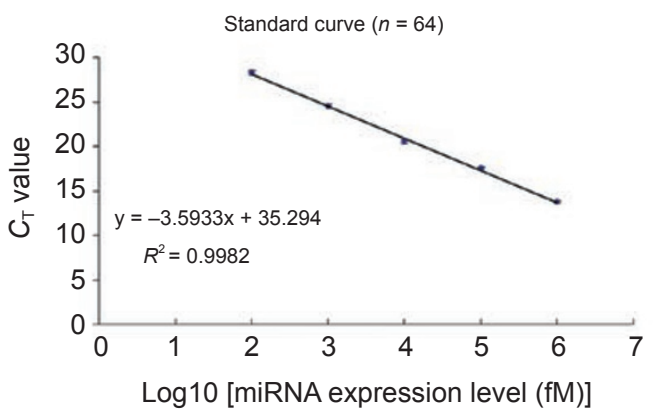

$\mathrm{C}$

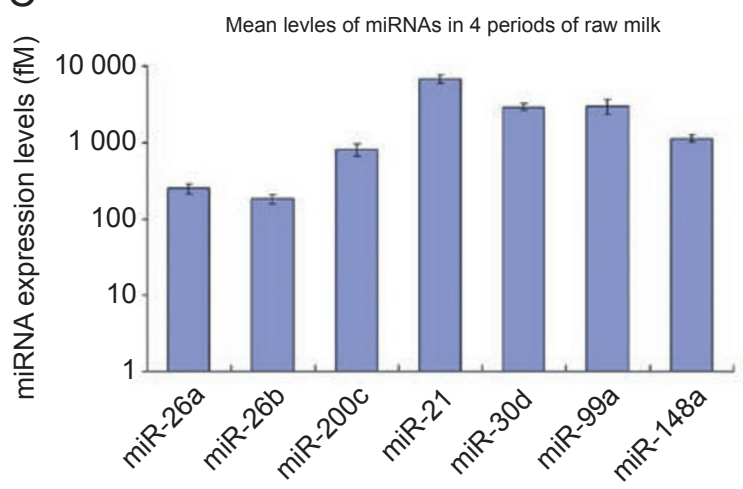

$E$

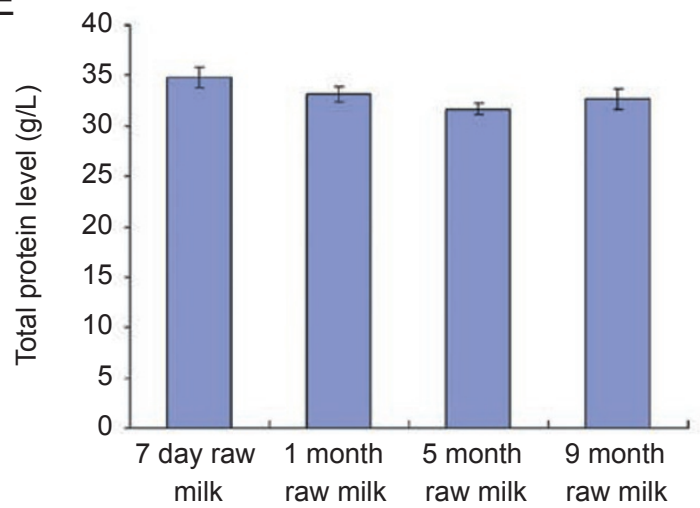

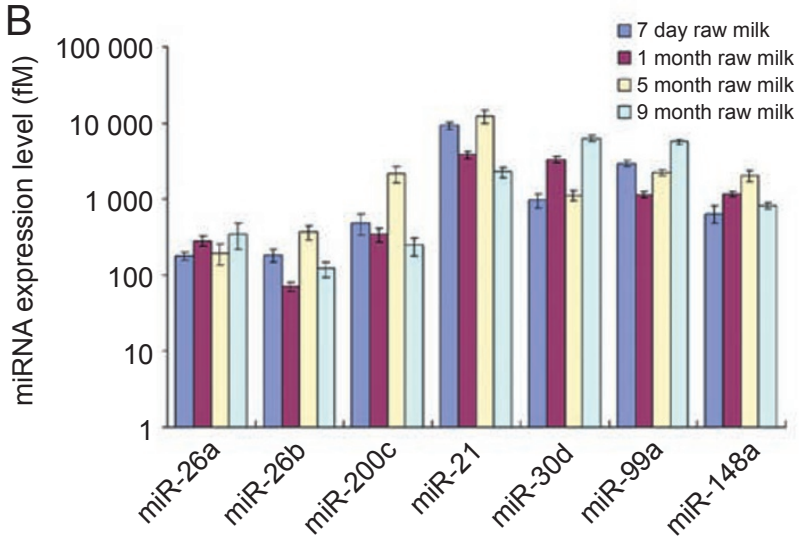

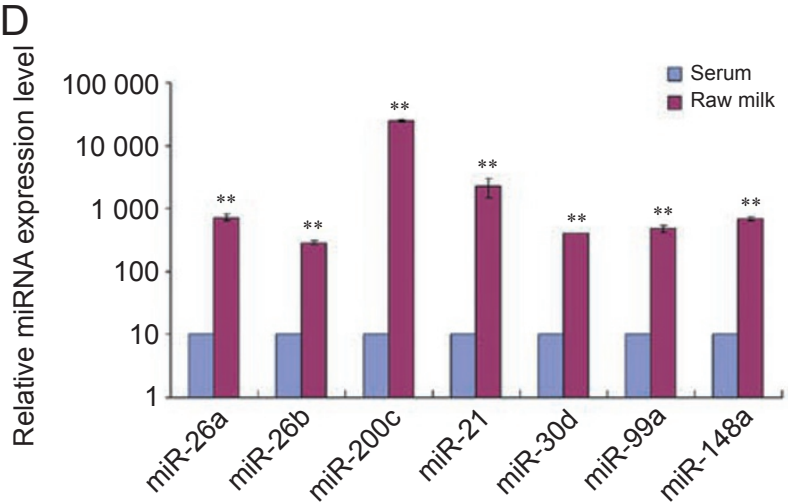

Figure 2 Consistent expression of the seven miRNAs in the milk from different stages of lactation. (A) Construction of standard curves. A series of synthetic miRNA oligonucleotides (Dalian, China) of known concentrations were reverse-transcribed and amplified. The miRNA levels were assessed by qRT-PCR assay. The resulting $C_{\mathrm{T}}$ values were plotted vs. the $\log _{10}$ of the amount of input miRNAs. (B) Comparison of the expression levels of the seven miRNAs in the raw milk collected at day 7 ( $n$ $=20)$, month $1(n=20)$, month $5(n=20)$, and month $9(n=20)$ of lactation. The absolute amount of each miRNA in the milk from different stages of lactation was assessed by qRT-PCR assay and calculated by referring to the standard curve. (C) The mean expression levels of the seven miRNAs in the raw milk collected at day $7(n=20)$, month $1(n=20)$, month $5(n=20)$, and month $9(n=20)$ of lactation were used to represent the general miRNA levels in raw milk. (D) Comparison of the expression levels of the seven miRNAs in raw milk $(n=9)$ and serum $(n=8)$. The miRNA expression levels were determined by qRT-PCR. For comparison, the expression levels of miRNAs in serum were arbitrarily set at 10 . The $y$ axes showed arbitrary units representing relative miRNA expression levels. Results are presented as means \pm SEM of three independent experiments $\left({ }^{*} P<0.05, * * P<0.01\right)$. (E) Comparison of the total protein levels in the raw milk collected at day $7(n=20)$, month 1 ( $n$ $=20)$, month $5(n=20)$, and month $9(n=20)$ of lactation. The total protein levels were determined by BCA kit (Pierce, Rockford, IL, USA). 
For the expression profile of seven miRNAs to be an indicator for milk content, it must specifically and quantitatively reflect the alteration of milk content. Next, we tested the sensitivity and specificity of the milk miRNAbased assay in detecting the raw milk content. For this assay, raw milk was serially diluted with water to a final concentration of $100 \%, 90 \%, 80 \%, 70 \%, 60 \%, 50 \%$, or $40 \%$, respectively, and the expression levels of the seven miRNAs in each diluted milk sample were analysed by miRNA qRT-PCR. As shown in Figure 3A, the diluted
A

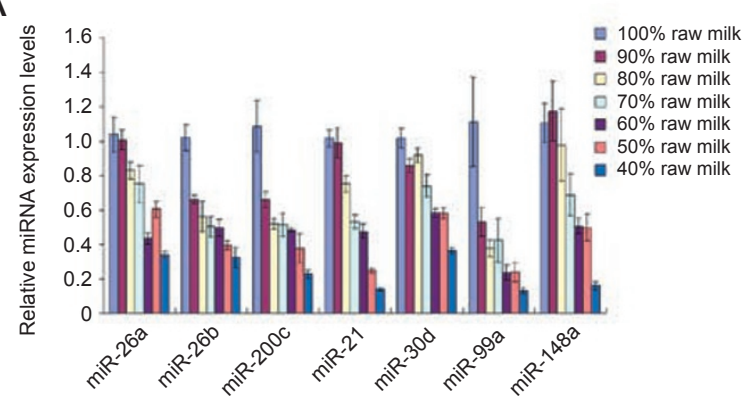

C

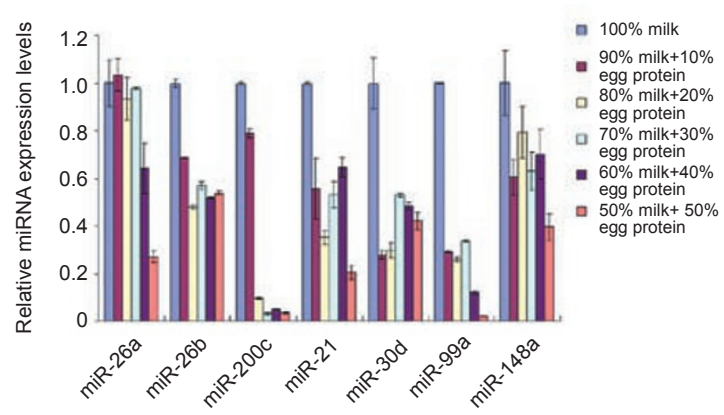

E

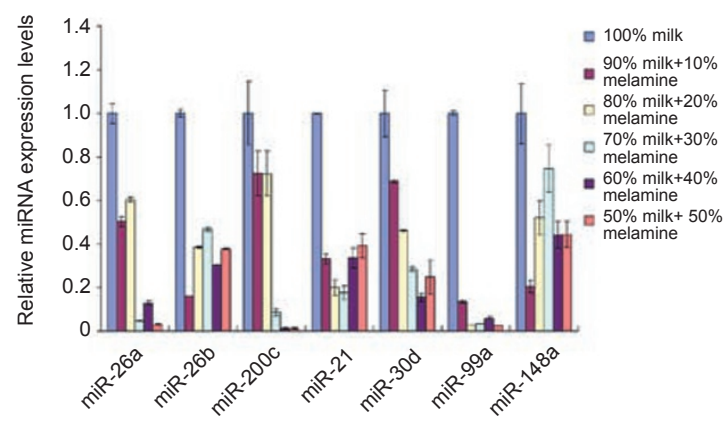

B

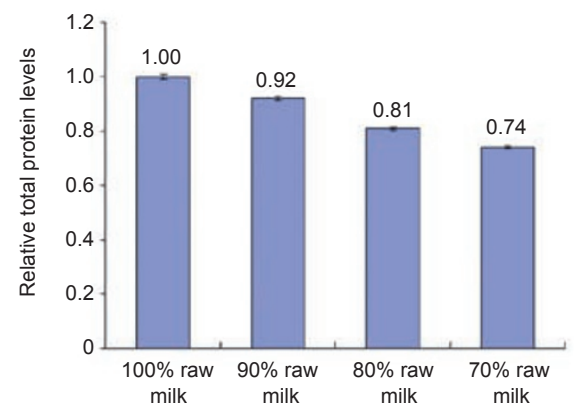

D

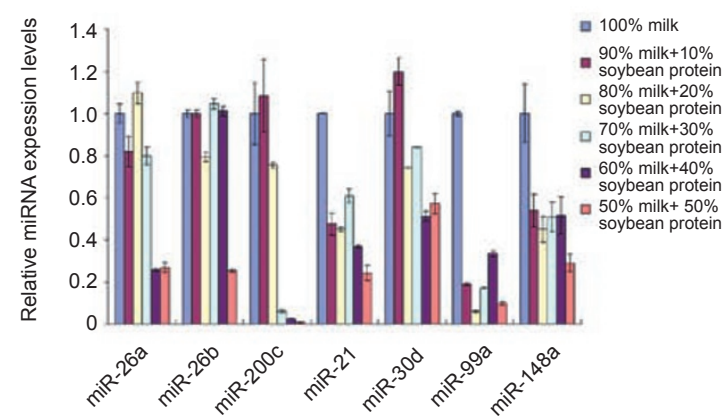

$\mathrm{F}$

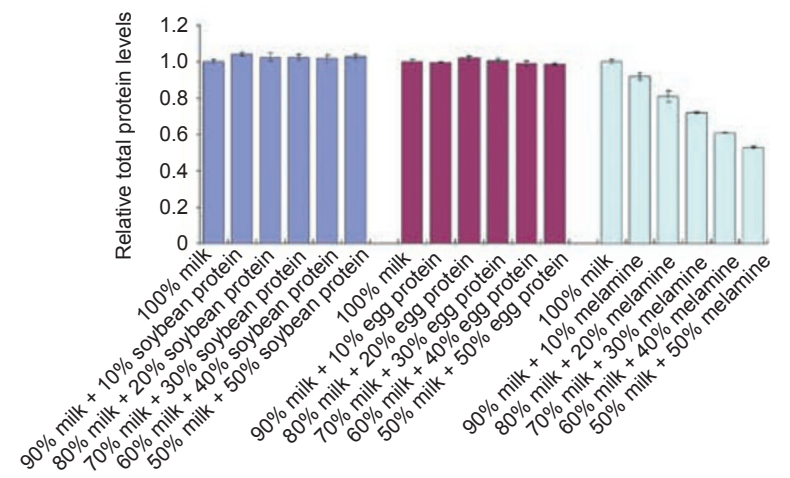

Figure 3 Expression profile of the seven miRNAs as a biomarker for distinguishing the manipulated milk from pure raw milk. (A, B) Raw milk samples were serially diluted (with water) to a final concentration of $100 \%, 90 \%, 80 \%, 70 \%, 60 \%, 50 \%$, or $40 \%$, respectively. The expression levels of the seven miRNAs in each diluted milk sample were analysed by qRT-PCR, and the total protein levels were determined by using a BCA kit. For comparison, the expression levels of miRNAs and total protein in $100 \%$ raw milk were arbitrarily set at 1 . The $y$ axes showed arbitrary units representing relative miRNA expression levels. (CF) Using egg protein or soybean protein, solutions whose protein concentration was equal to that of raw milk were prepared. Likewise, solutions whose nitrogen concentration were equal to that of raw milk were prepared by using melamine. Diluted milk samples were supplemented with these solutions to elevate their total protein or nitrogen levels to the level of raw milk. The expression levels of the seven miRNAs in each manipulated milk sample were analysed by qRT-PCR. The total protein levels were determined by BCA kit. For comparison, the expression levels of miRNAs and total protein in $100 \%$ raw milk were arbitrarily set at 1 . The $y$ axes show arbitrary units representing relative miRNA expression levels. Results are presented as means \pm SEM of three independent experiments $(* P<0.05$, $* * P<0.01)$. 
A

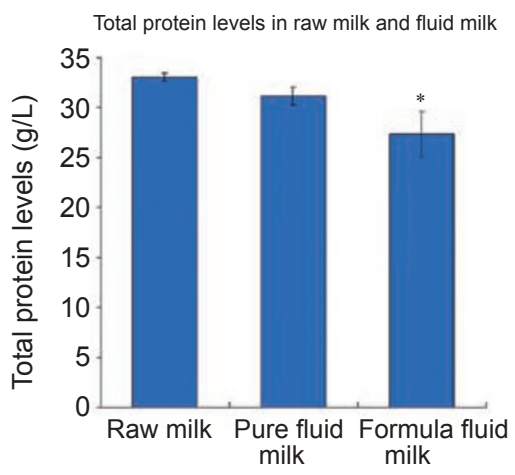

C
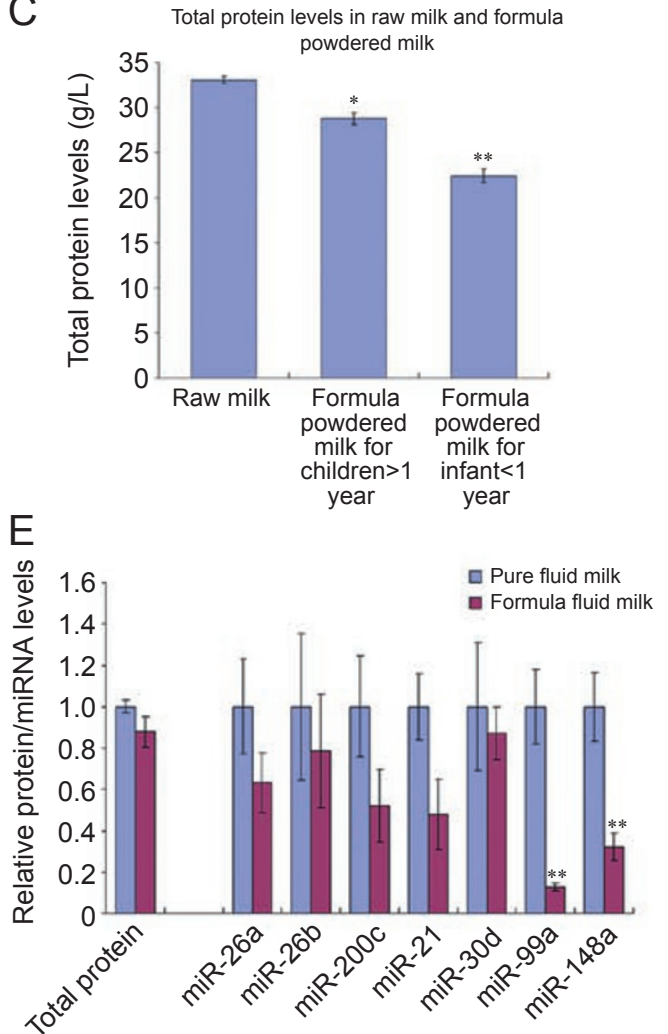

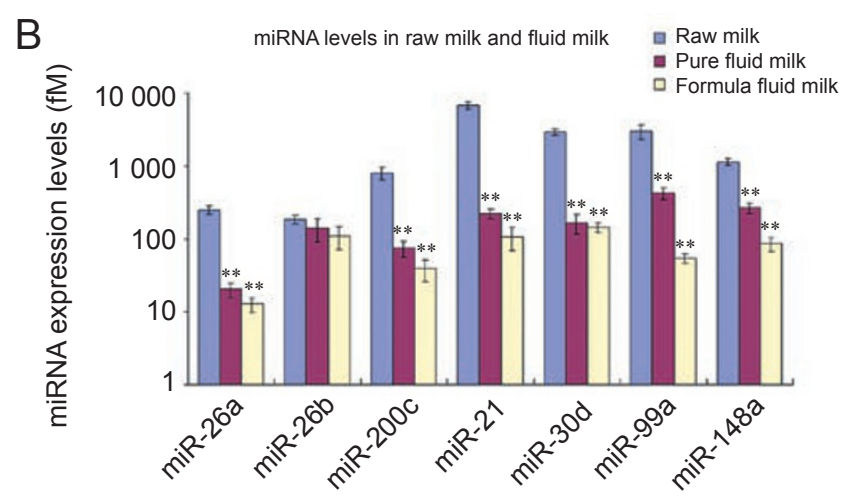

$\mathrm{D}$

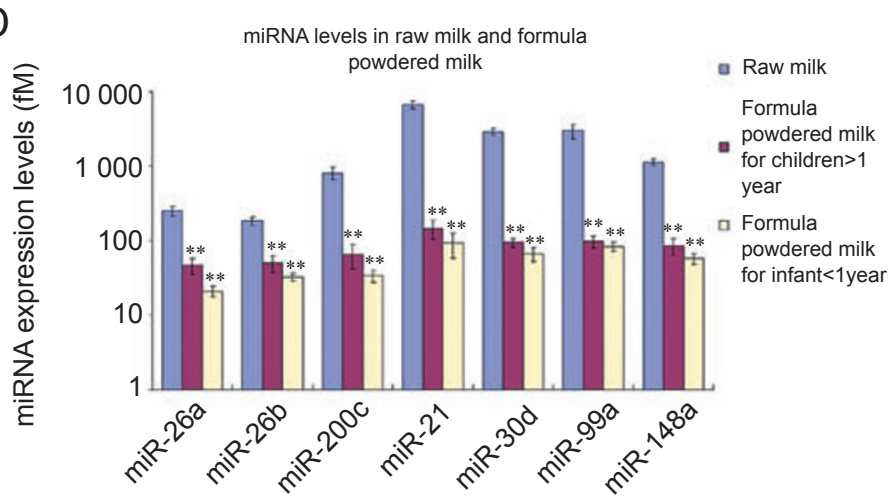

F

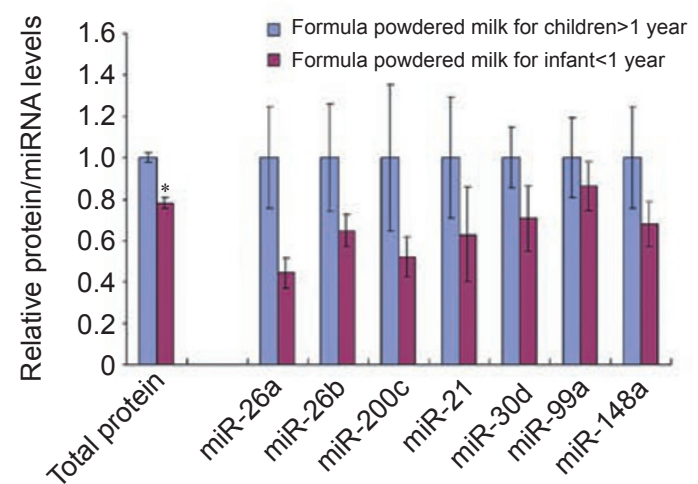

Figure 4 Expression profile of the seven miRNAs as a biomarker for the quality control of commercial milk products. (A) Comparison of the total protein levels in pure fluid milk $(n=11)$, formula fluid milk $(n=14)$, and raw milk $(n=80)$. The total protein levels were determined by BCA kit. (B) Comparison of the expression levels of the seven miRNAs in pure fluid milk $(n=11)$, formula fluid milk $(n=14)$, and raw milk $(n=80)$. The absolute amount of each miRNA was assessed by qRT-PCR assay and calculated by referring to the standard curve. (C) Comparison of the total protein levels in the formula powdered milk for children (>1 year) $(n=14)$, the formula powdered milk for infant (<1 year) $(n=11)$, and raw milk $(n=80)$. The total protein levels were determined by BCA kit. (D) Comparison of the expression levels of the seven miRNAs in the formula powdered milk for children (>1 year) $(n=14)$, the formula powdered milk for infant $(<1$ year) $(n=11)$, and raw milk $(n=80)$. The absolute amount of each miRNA was assessed by qRTPCR assay and calculated by referring to the standard curve. (E) Comparison of the expression levels of the total protein and the seven miRNAs in pure fluid milk $(n=11)$ and formula fluid milk $(n=14)$. (F) Comparison of the expression levels of the total protein and the seven miRNAs in the formula powdered milk for children ( $>1$ year) $(n=14)$ and the formula powdered milk for infant $(<1$ year) $(n=11)$. Results are presented as means \pm SEM of three independent experiments $(* P<0.05 ; * * P<0.01)$.

milk samples showed a parallel reduction of the expression levels of the seven miRNAs. Likewise, the serial dilution of milk samples also resulted in decrease in total protein levels accordingly (Figure 3B). However, when diluted milk samples were supplemented with egg protein or soybean protein to elevate the total protein 
levels to that of raw milk (Figure 3F), these samples still showed significantly lower levels of the seven miRNAs (Figure 3C-3E). In diluted milk in which a pinch of melamine was added, expression levels of seven miRNAs remained low while the total nitrogen levels were brought to an equal level of raw milk (data not shown). These results clearly demonstrate that the expression profile of the seven milk-specific miRNAs can be an ideal biomarker for discriminating sub-standard or manipulated milk from raw milk.

Seven milk-specific miRNAs as a marker for quality control of milk-related products

Using the profile of seven miRNAs as a marker, we further assessed the quality of various commercial milk products. Among the 25 brands of fluid milk we surveyed, although the protein levels of fluid milk and formula fluid milk were similar to those of raw milk (Figure 4A), the amount of the seven miRNAs in fluid milk and formula fluid milk was significantly lower than that in raw milk (Figure 4B). We also surveyed 25 brands of formula powdered milk and found that the protein amount was only slightly lower in the formula powdered milk for children ( $>1$ year) and the formula powdered milk for infants $(<1$ year), compared to that in raw milk (Figure $4 \mathrm{C}$ ). In contrast, the expression levels of the seven miRNAs in those formula powdered milk were sharply lower (Figure 4D). Figure 4E and 4F also showed that, although the protein levels in each sample appeared the same, the expression levels of seven miRNAs were significantly reduced in formula fluid milk compared to pure fluid milk, and in formula powdered milk for infants $(<1$ year) compared to formula powdered milk for children ( $>1$ year), respectively. The results clearly indicate that the protein levels of these fluid milk and formula powdered milk are at least partially contributed by some proteins with sources other than raw milk.

\section{Discussion}

A joint declaration by the World Health Organization (WHO) and the United Nations Children's Fund (UNICEF) stated that breast milk is the optimal food for infants and can never be equalled by artificial substitutes [1]. It covers most of children's physiological and nutritional needs during their first 4-6 months of life [1]. Therefore, there is growing interest in attempting to make infant formulas more closely resembling breast milk. Previous studies have shown that extracellular nucleic acids, both DNA and RNA, are present in milk and have important physiological roles in breast-fed infants $[31,32]$. They act not only as metabolites but also as bio- active substances in regulating body functions [31, 32]. In the present study, we further demonstrate that miRNA is a natural inherent component of cow milk and is stably present in milk. Although it is unclear whether miRNAs in milk originate from the active secretion by mammary gland or the passive leak by mammary gland cells, the expression profile of milk miRNA is significantly different at various lactation stages. The findings strongly implicate that milk miRNAs may be involved in the regulation of cow lactation and also have different biological functions in the processes of new-born calf organ or immune system development. The finding of miRNAs in cow milk suggests that breast milk may also possess miRNAs and further research should be conducted to determine what role these miRNAs play in human development and whether they should be added to infant formula. Indeed, very recently Kosaka et al. reported that breast milk contains miRNAs and these miRNAs may have potential biological functions in modulating cell growth and diffentiation [33]. Hata et al. also showed that a considerable amount of mammary gland and immune-related miRNAs were present in the milk-derived microvesicles [34]. By comparing the miRNA profiling results obtained in cow milk and breast milk, we found that the majority of miRNAs, especially the immunespecific ones, are quite similar in cow milk and breast milk. Thus, our results, together with those of others [33, 34], may serve as a starting point for future studies investigating if intestinal health and baby performance can be improved by introducing dietary miRNAs in infant diets during the before- and post-weaning period.

More importantly, through a systematic analysis of milk miRNAs at various lactation stages, we have identified seven miRNAs that are stably present in milk and their levels can serve as an internal "standard" or "biomarker" for quality control of milk. This miRNA-based novel biomarker can accurately reflect various aspects of milk quality, which in turn can reveal the actual content of raw milk in the "manipulated" milk or milk with poor quality. The identification of miRNAs as novel ingredients and quality indicators of raw milk will have a great potential in revolutionising the entire dairy industry, particularly the area of milk quality control.

\section{Materials and Methods}

\section{Milk sample and serum sample preparation}

A farm in eastern China was selected for raw milk sampling. This farm is equipped with modern automated milking facilities, and its milk products are considered to be high grade according to the national quality standards of China. There is no grazing area in this farm, and the herd's nutrition is based on a feeding mixture that is prepared in a central regional feeding centre. All cows are 
in perfectly healthy condition.

Raw milk samples were taken under sterile conditions from a mix of multiple milking products. Particularly, 40 cows at 9 months postpartum and 40 cows at 7 days postpartum were recruited, and $100 \mathrm{ml}$ of mature milk (taken from cows at 9 months postpartum) or colostrums (taken from cows at 7 days postpartum) were collected, respectively. Milk samples were transported to the laboratory on ice and centrifuged at $1500 \times g$ for $20 \mathrm{~min}$ to remove the cell debris. The supernatant, milk plasma, was recovered and stored at $-80{ }^{\circ} \mathrm{C}$ for further analysis.

Blood samples were collected from 40 cows ( $5 \mathrm{ml}$ each) and placed in a serum separator tube. Samples were processed without delay. Separation of the serum was accomplished by centrifugation at $800 \times g$ for $10 \mathrm{~min}$, followed by a 15 -min high-speed centrifugation at $10000 \times g$ to completely remove the cell debris. The supernatant serum was recovered and stored at $-80{ }^{\circ} \mathrm{C}$ until analysis.

\section{Solexa sequencing}

The sequencing procedure was conducted as previously described [7, 15]. Briefly, $2.5 \mathrm{ml}$ mature milk, colostrum, or serum from each cow was pooled together. Total RNA was extracted from $100 \mathrm{ml}$ of mature milk, colostrum, or serum using the Trizol LS Reagent (Invitrogen, Carlsbad, CA, USA), according to the manufacturer's instructions. After the PAGE purification of small RNA molecules (under 30 bases) and the ligation of a pair of Solexa adaptors to their 5' and $3^{\prime}$ ends, the small RNA molecules were amplified using the adaptor primers for 17 cycles, and the fragments of around $90 \mathrm{bp}$ (small RNA + adaptors) were isolated from agarose gels. The purified DNA was directly used for the cluster generation and sequencing analysis using an Illumina Genome Analyzer, according to the manufacturer's instructions. The image files generated by the sequencer were then processed to produce digital-quality data. After masking the adaptor sequences and removing the contaminated reads, clean reads were processed for computational analysis.

Solexa reads were aligned against the bovine genome by SOAP. Sequences with perfect match or one mismatch were retained for further analysis. To further analyse the RNA secondary structure comprising the matched Solexa reads, $100 \mathrm{nt}$ of genomic sequence flanking each side of these sequences were extracted, and the secondary structure was predicted using RNAfold and analysed by MIREAP under default settings. MIREAP is a computational tool specially designed to identify novel miRNAs from deeply sequenced small RNA libraries, fully taking into consideration the miRNA biogenesis, sequencing depth, and structural features to improve the sensitivity and specificity of miRNA identification. Stem-loop hairpins were considered typical only when they fulfilled all the three criteria: (a) mature miRNAs are present in one arm of the hairpin precursors lacking large internal loops or bulges; (b) the secondary structures of the hairpins are steady, with the free energy of hybridisation being lower than $-20 \mathrm{kcal} /$ mol; (c) hairpins are located in intergenic regions or introns. Those genes whose sequences and structures fulfilled all of these criteria were considered as candidate miRNA genes. Finally, the candidate miRNA reads were used as queries in the BLAST searches of the miRBase database 13.0, tolerating two mismatches.

For comparison of the expression levels of miRNAs between mature milk and colostrum, the reads of individual miRNAs were subjected to the analysis as follows: a miRNA was considered "altered" only if it had at least: (a) 20 copies by Solexa sequencing in either mature milk or colostrums, and (b) two-fold expression alteration between mature milk and colostrum.

\section{qRT-PCR assay}

Total RNA was extracted from $100 \mu \mathrm{l}$ milk or serum by phenol/ chloroform purification and isopropyl alcohol precipitation. Briefly, $100 \mu \mathrm{l}$ milk was mixed with $200 \mu \mathrm{l}$ phenol, $200 \mu \mathrm{l}$ chloroform, and $400 \mu \mathrm{l}$ Rase-free $\mathrm{H}_{2} \mathrm{O}$. The mixture was vortexed and kept at room temperature for $15 \mathrm{~min}$. The mixture was centrifuged at 12 $000 \times g$ for $15 \mathrm{~min}$, and the upper (aqueous) phase was collected. Subsequently, a $1 / 10$ volume of $3 \mathrm{~mol} / \mathrm{L}$ sodium acetate and a twofold volume of isopropyl alcohol were added to the aqueous phase; the total RNA was precipitated by keeping the sample at $-20{ }^{\circ} \mathrm{C}$ for $1 \mathrm{~h}$. RNA pellet was collected by centrifuging at $12000 \times \mathrm{g}$ for 20 min. RNA pellet was washed once with $75 \%$ ethanol, dried for $10 \mathrm{~min}$ at room temperature, and then dissolved in $20 \mu \mathrm{l}$ of RNasefree water.

qRT-PCR was carried out using TaqMan microRNA probes (Applied Biosystems, Foster City, CA, USA), according to the manufacturer's instructions [29, 30]. Briefly, $5 \mu$ l of total RNA was reverse-transcribed to cDNA using AMV reverse transcriptase (TaKaRa, Dalian, China) and stem-loop RT primers (Applied Biosystems, Foster City, CA, USA). Real-time PCR was performed using a TaqMan PCR kit on an Applied Biosystems 7300 Sequence Detection System (Applied Biosystems, Foster City, CA, USA). All reactions, including the no-template controls, were run in triplicate. After the reactions, the $C_{\mathrm{T}}$ values were determined using fixed-threshold settings. To calculate the absolute expression levels of the target miRNAs, a series of synthetic miRNA oligonucleotides of known concentrations were also reverse-transcribed and amplified. The absolute amount of each miRNA was then calculated by referring to the standard curve.

\section{Statistical analysis}

qRT-PCR assay was performed in triplicate, and the entire experiment was repeated multiple times. Data shown were presented as means \pm SEM of three or more independent experiments, and the differences were considered statistically significant at $P<0.05$ by Student's $t$-test.

\section{Acknowledgments}

This work was supported by grants from the National Natural Science Foundation of China (30225037, 30471991, 30570731), the 973 Program of China (2006CB503909, 2004CB518603), the "111" Project, and the Natural Science Foundation of Jiangsu Province (BK2004082, BK2006714).

\section{References}

1 Sobti J, Mathur GP, Gupta A. WHO's proposed global strategy for infant and young child feeding: a viewpoint. J Indian Med Assoc 2002; 100:502-504, 506.

2 Guan N, Fan Q, Ding J, et al. Melamine-contaminated powdered formula and urolithiasis in young children. $N$ Engl $J$ Med 2009; 360:1067-1074.

3 Ding J. Childhood urinary stones induced by melamine-tainted formula: how much we know, how much we don't know. 
Kidney Int 2009; 75:780-782.

4 Ambros V. The functions of animal microRNAs. Nature 2004; 431:350-355.

5 Bartel DP. MicroRNAs: genomics, biogenesis, mechanism, and function. Cell 2004; 116:281-297.

6 He L, Hannon GJ. MicroRNAs: small RNAs with a big role in gene regulation. Nat Rev Genet 2004; 5:522-531.

7 Chen X, Ba Y, Ma L, et al. Characterization of microRNAs in serum: a novel class of biomarkers for diagnosis of cancer and other diseases. Cell Res 2008; 18:997-1006.

8 Mitchell PS, Parkin RK, Kroh EM, et al. Circulating microRNAs as stable blood-based markers for cancer detection. Proc Natl Acad Sci USA 2008; 105:10513-10518.

9 Gilad S, Meiri E, Yogev Y, et al. Serum microRNAs are promising novel biomarkers. PLoS ONE 2008; 3:e3148.

10 Resnick KE, Alder H, Hagan JP, et al. The detection of differentially expressed microRNAs from the serum of ovarian cancer patients using a novel real-time PCR platform. Gynecol Oncol 2009; 112:55-59.

11 Park NJ, Zhou H, Elashoff D, et al. Salivary microRNA: discovery, characterization, and clinical utility for oral cancer detection. Clin Cancer Res 2009; 15:5473-5477.

12 Hanke M, Hoefig K, Merz H, et al. A robust methodology to study urine microRNA as tumor marker: microRNA-126 and microRNA-182 are related to urinary bladder cancer. Urol Oncol 2009 Apr 16. doi:10.1016/j.urolonc.2009.01.027

13 Skog J, Wurdinger T, van Rijn S, et al. Glioblastoma microvesicles transport RNA and proteins that promote tumour growth and provide diagnostic biomarkers. Nat Cell Biol 2008; 10:1470-1476.

14 Valadi H, Ekstrom K, Bossios A, et al. Exosome-mediated transfer of mRNAs and microRNAs is a novel mechanism of genetic exchange between cells. Nat Cell Biol 2007; 9:654659.

15 Chen X, Li Q, Wang J, et al. Identification and characterization of novel amphioxus microRNAs by Solexa sequencing. Genome Biol 2009; 10:R78.

16 Griffiths-Jones S. The microRNA Registry. Nucleic Acids Res 2004; 32:D109-D111.

17 Sonkoly E, Stahle M, Pivarcsi A. MicroRNAs and immunity: novel players in the regulation of normal immune function and inflammation. Semin Cancer Biol 2008; 18:131-140.

18 Sempere LF, Freemantle S, Pitha-Rowe I, et al. Expression profiling of mammalian microRNAs uncovers a subset of brain-expressed microRNAs with possible roles in murine and human neuronal differentiation. Genome Biol 2004; 5:R13.

19 Wienholds E, Kloosterman WP, Miska E, et al. MicroRNA expression in zebrafish embryonic development. Science 2005; 309:310-311.
20 Beuvink I, Kolb FA, Budach W, et al. A novel microarray approach reveals new tissue-specific signatures of known and predicted mammalian microRNAs. Nucleic Acids Res 2007; 35:e52.

21 Shingara J, Keiger K, Shelton J, et al. An optimized isolation and labeling platform for accurate microRNA expression profiling. $R N A$ 2005; 11:1461-1470.

22 Liang Y, Ridzon D, Wong L, Chen C. Characterization of microRNA expression profiles in normal human tissues. $B M C$ Genomics 2007; 8:166.

23 Schratt GM, Tuebing F, Nigh EA, et al. A brain-specific microRNA regulates dendritic spine development. Nature 2006; 439:283-289.

24 Ryan DG, Oliveira-Fernandes M, Lavker RM. MicroRNAs of the mammalian eye display distinct and overlapping tissue specificity. Mol Vis 2006; 12:1175-1184.

25 Szafranska AE, Davison TS, John J, et al. MicroRNA expression alterations are linked to tumorigenesis and non-neoplastic processes in pancreatic ductal adenocarcinoma. Oncogene 2007; 26:4442-4452.

26 Landgraf P, Rusu M, Sheridan R, et al. A mammalian microRNA expression atlas based on small RNA library sequencing. Cell 2007; 129:1401-1414.

27 Wang S, Aurora AB, Johnson BA, et al. The endothelialspecific microRNA miR-126 governs vascular integrity and angiogenesis. Dev Cell 2008; 15:261-71.

28 Masaki S, Ohtsuka R, Abe Y, Muta K, Umemura T. Expression patterns of microRNAs 155 and 451 during normal human erythropoiesis. Biochem Biophys Res Commun 2007; 364:509-514.

29 Chen C, Ridzon DA, Broomer AJ, et al. Real-time quantification of microRNAs by stem-loop RT-PCR. Nucleic Acids Res 2005; 33:e179.

30 Tang F, Hajkova P, Barton SC, Lao K, Surani MA. MicroRNA expression profiling of single whole embryonic stem cells. Nucleic Acids Res 2006; 34:e9.

31 Semenov DV, Kuligina EV, Shevyrina ON, Richter VA, Vlassov VV. Extracellular ribonucleic acids of human milk. Ann N Y Acad Sci 2004; 1022:190-194.

32 Kuligina EV, Semenov DV, Shevyrina ON, Richter VA. Ribonucleic acids of human milk. Nucleosides Nucleotides Nucleic Acids 2004; 23:837-842.

33 Kosaka N, Izumi H, Sekine K, Ochiya T. microRNA as a new immune-regulatory agent in breast milk. Silence 2010; 1:7.

34 Hata T, Murakami K, Nakatani H, Yamamoto Y, Matsuda T, Aoki N. Isolation of bovine milk-derived microvesicles carrying mRNAs and microRNAs. Biochem Biophys Res Commun 2010 Apr 29. doi:10.1016/j.bbrc.2010.04.135

(Supplementary information is linked to the online version of the paper on the Cell Research website.) 